\title{
An Auctioning Mechanism for Green Radio
}

\author{
Cristina Comaniciu, Narayan B. Mandayam, H. Vincent Poor, and Jean-Marie Gorce
}

\begin{abstract}
In this paper, an auctioning strategy is proposed for cellular networks that ensures net energy savings. The pricing scheme, in conjunction with a two dimensional bid structure, incentivizes cooperation at the terminal nodes for better interference management at receivers and for cooperative relaying. It is shown that, for the proposed auctioning strategy, network operators are guaranteed revenue gains, mobile nodes' dominant strategy is to bid their true valuation of their energy resources, and overall effective energy gains occur under the assumption of a reserve price for bidding. Simulation results show that significant energy savings can be achieved by employing this auctioning mechanism for a 3G cellular set-up.
\end{abstract}

Index Terms: Cellular, cooperative relaying, green radio, interference management, pricing, Vickrey auction.

\section{INTRODUCTION}

Climatologists have determined that the accumulation of greenhouse gases (primarily carbon dioxide- $\mathrm{CO}_{2}$ ) in the upper levels of the earth's atmosphere is forming a shield that prevents heat rays escaping from the earth, thereby warming the planet to unnatural levels [1], [2]. Scientists have warned that such global warming may have significant negative impact on global health, social and economic well-being. One measure that objectively quantifies the impact of modern civilization on global warming is the carbon footprint, which measures the quantity (in pounds or kilograms) of $\mathrm{CO}_{2}$ released into the atmosphere by a certain human activity. Greenhouse gas (GHG) emissions have risen sharply with the industrial revolution and they are projected to further increase dramatically if significant measures are not taken to prevent this. Currently, the telecommunication industry sector accounts for about $2 \%$ of the GHG footprint (about the same percentage as air traffic) [3]. For the wireless communications, most of the significant environmental impact is due to high energy consumption in cellular base stations (BSs) [4].

There is considerable current interest in reducing the energy consumption in the wireless sector, particularly in Europe, with companies such as Orange (France), Erricson (Sweden), and Vodaphone (UK) vouching to dramatically reduce their $\mathrm{CO}_{2}$ emissions in the range of $50-80 \%$ by 2020 [4]. Further, significant effort has been directed toward the design of base stations powered by alternate energy sources (e.g., solar cells and wind tur-

Manuscript received October 1, 2009.

This paper was presented in part at IEEE Vehicular Technology Conference, Anchorage, Alaska, September 2009.

C. Comaniciu is with the ECE Department, Stevens Institute of Technology, USA, email: ccomanic@stevens.edu.

N. B. Mandayam is with WINLAB, Rutgers University, USA, email: narayan@winlab.rutgers.edu.

H. V. Poor is with the Electrical Engineering Department, Princeton University, USA, email: poor@princeton.edu.

J.-M. Gorce is with Université de Lyon, INRIA INSA-Lyon, CITI, F-69621, France, email: jean-marie.gorce@insa-lyon.fr. bines).

To better understand the impact of energy consumption for wireless networks, consider the example discussed in [5] of a typical 3G operator in Europe who generates approximately 11 tons of $\mathrm{CO}_{2}$ from each cell site each year. In [5], the authors conjecture that a $34 \%$ reduction in the overall energy consumption for the operator would save it $\$ 25$ million each year, while reducing the $\mathrm{CO}_{2}$ footprint by about 88,600 tons per year. Although research on the design of green radio solutions is still in its infancy, significant results already exist on energy efficient designs to conserve battery power for mobile terminals and wireless sensors. Some of the prior work on green wireless also focuses on reducing the transmission power at the mobile terminals, in order to reduce potential harmful effects to users' health [6]. Because of the focus on mobile terminals, most of the prior work on energy efficiency has primarily addressed uplink communications. However, from an overall perspective, it is necessary to consider the downlink, as base stations are the primary energy consumers of cellular networks. Consequently, in this paper, we approach energy efficiency from the perspective of optimized downlink communications, with a direct objective of reducing energy consumption at the base station. Very few papers on green communications address the downlink problem, with some prior work focusing on cell site dimensioning [7], [8], and improving the efficiency of power amplifiers in the base stations [9].

Following up on the idea that smaller cell sites may result in lower transmission energy for base stations, but with a drawback of increasing their overall number in the network, our approach is to reduce the required transmission range for the base station by exploiting relaying by mobile terminals.

We conjecture that cooperation between end users and network operators may result in significant overall energy savings. We propose an auction based pricing system that incentivizes both parties to cooperate for energy savings, and we prove that a game theoretic model for this auction admits a Nash equilibrium at which the network operator rewards cooperative behavior of mobile terminals, while the terminals have a dominant strategy of bidding their true valuation of energy resource.

In this formalism, the nodes' cooperative options include employing better signal processing/interference cancellation techniques, flexible QoS requirements, and relaying packets for remote nodes.

While our analysis shows that effective energy gains can be achieved by the proposed auctioning scheme, the magnitude of the gains can only be quantified via simulation results. These results enhance our earlier conference publication in [10], which has focused only on the theoretical analysis.

Our preliminary simulation results show that energy gains as high as 50\% can be achieved in a 3G CDMA cell. Actual gains depend on the system load, mobile terminals' density, distance of the destination nodes from the base station, and the actual 
pricing scheme employed.

\section{ENERGY CONSUMPTION MODELS}

\section{A. Energy based Utility Models for Base Station}

According to references [4] and [11], a significant part of the energy consumption for network operators is the operating energy for the base stations (as opposed to the embodied energy for manufacturing, as it is in the case for mobile terminals), with about $57 \%$ of the energy consumption being used for radio access. It is thus apparent that efficient resource management algorithms are key components for more efficient communications.

By analyzing the base station power model consumption in [11], we can see that significant energy is consumed in transmit power, which results in even higher energy consumption due to the inefficiency of the linear power amplifiers (LPAs). Consequently, according to [11], for each watt of output power, approximately $3.5 \mathrm{~W}$ are required at the LPA input. Given this, the effective gains in reducing output power at the base station need to be multiplied by the inverse of the LPA efficiency at the base station, $\eta_{B}$.

As $3 \mathrm{G}$ services mature, the revenue of mobile network operators is expected to grow at a much slower rate than the corresponding increase in the amount of data traffic [4], with the starkest difference seen so far with the companies that offer more aggressive pricing for data services [4]. One way to improve revenues for the operators is to reduce their energy costs, so that becoming "greener" for environmental reasons also becomes financially beneficial.

Assuming an effective cost per unit energy to be $c$, the cost of transmitting 1 bit for a connection can be expressed as (similarly to the energy per bit utility function for mobile terminals proposed in [12]):

$$
C_{b}=\frac{\eta_{B} P_{t} c E[K]}{r}=\frac{\eta_{B} P_{t} c}{r P_{c}(\gamma)}
$$

where $r$ is the transmission rate, $E[K]=$ average number of required retransmissions per packet, and $P_{c}(\gamma)$ is the probability of correct reception of a packet, with $\gamma$ being the signalto-interference-plus-noise ratio (SINR) at the receiving node. $P_{c}(\gamma)$ is determined by the receiving node characteristics (e.g., current interference and noise levels, interference cancellation capabilities, etc.) and on the node's QoS specifications (e.g., transmission rate, modulation level, etc.).

The cost in (1) can be reduced by:

i. Better interference management at receiving nodes.

ii. Reduced QoS requirements in terms of transmission rates.

iii. Support of external terminals for relaying, with an effect of reducing $P_{t}$, by using a smaller effective transmission range for the base station.

From the above discussion, it is apparent that cooperation from mobile terminals may potentially result in gains for the network operators. One way to incentivize such cooperation is to introduce reward pricing for cooperating nodes (see for example [13]). These rewards will encourage the nodes to trade off their QoS requirements or energy (for the case of relaying nodes) for monetary rewards from the system operator. We note that in designing such a pricing scheme, the green energy savings goal is to reduce the overall energy consumption per transmitted bit, rather than simply move the energy burden from the base station (BS) to the mobile terminals.

The network operator's utility per transmitted bit is proportional to the revenue obtained from the transmission. A higher transmission power will result in higher energy costs, thus diminishing the overall revenue of the operator. The revenue of the operator comes from the price per bit $\lambda$ charged to the end user. A reimbursement price per bit $R$ can be used to reward intermediate nodes that facilitate transmission with reduced energy costs. Thus, the utility function perceived by the base station operators is a measure of revenue per bit and can be expressed as (see also [13] for a similar definition):

$$
U_{\mathrm{BS}}=\lambda-E_{b} c-R
$$

where $E_{b}$ is the energy per transmitted bit consumed by the BS: $E_{b}=\eta_{B}\left(P_{t}\right) P_{t} / r P_{c}(\gamma)$, and $R$ is the monetary reward for relaying which could be expressed as $R=E_{b}^{\prime} \mu$, with $E_{b}^{\prime}=$ energy per bit consumed by a relaying node, and $\mu=$ unit price per energy expenditure.

We note that $E_{b}$ in (2) may represent the energy spent for sending directly to the destination node (direct link - no relay), or the energy spent to send to the relaying node (in case of cooperative relaying).

Based on the above discussion the BS utility function can be written as

$$
U_{\mathrm{BS}}=\lambda-E_{b} c-E_{b}^{\prime} \mu .
$$

We note that a profit margin $\epsilon$ per unit energy expenditure for the BS could be defined, such as to guarantee the network operator's revenue in the absence of helper terminals $\epsilon=\lambda-$ $\left(E_{b} c\right)_{\max }>0$. To determine a fixed price $\lambda$ that will guarantee a positive profit margin, the network operator must estimate a worst case $\left(E_{b}\right)_{\max }$ for all potential connections.

\section{B. Utility Models for Mobile Terminals}

Two different terminal types should be considered for modeling: (i) The intended receiver (destination node); and (ii) a relaying terminal.

\section{B.1 Destination Node}

At the receiving node, the utility of the terminal measures the level of satisfaction a customer gets from the service it receives. A general valuation function $\Gamma\left(T_{D}, \lambda, q\right)$ is proposed to capture the following performance metrics that are relevant to the destination terminal: Throughput $\left(T_{D}\right)$, price charged by the BS per correctly received bit $(\lambda)$, and energy expenditure for advanced signal processing $(q)$. The function $\Gamma$ should be: (i) Monotonically increasing in throughput $T_{D}$; (ii) monotonically decreasing in $\lambda$ and $q$.

Consequently, the destination node's utility can be determined to be equal to its valuation function:

$$
U_{D}=\Gamma\left(T_{D}, \lambda, q\right)
$$

The selection of function $\Gamma$ will determine the relative preferences given by a user to these three metrics. Given the price 
$\lambda$ announced by the BS, and the user's profile parameters, the destination terminal optimizes its utility function, for a set of choices in the following areas: Signal processing algorithms for better interference management at the receiver, and QoS specifications in terms of throughput requirements for the connection.

The destination node can trade off required throughput for lower payment, or can choose to implement sophisticated signal processing at the receiver (e.g., multiuser detection, diversity, beamforming, etc.) to reduce the interference level with a direct impact in reducing the required transmission power for the base station (for a given SINR requirement). The latter choice comes with a price of increased computational energy expenditure, which also affects the terminal's utility.

The choice of the function $\Gamma$ is not unique. Future work will consider the impact of this function definition on capturing the real preferences of the destination user, and on impacting the achievable performance metrics at user and network level.

\section{B.2 Relaying Node}

Using relaying nodes could be beneficial for reducing the overall energy consumption of the transmission. The operator can reward cooperative behavior of the nodes, by paying a reimbursement price for the energy expended by relaying terminals.

The utility of a relaying terminal will depend on the reward gained $R$, and on the energy expenditure of the terminal $E_{b}^{\prime}$, through the valuation function $\theta\left(E_{b}^{\prime}, c\right)$ :

Definition 1. The valuation function $\theta\left(E_{b}^{\prime}, c\right)$ is the minimal reward price the potential relaying terminal would accept to be paid for its energy expenditure $E_{b}^{\prime}$.

An example of a choice for the valuation function for a user could be $\theta\left(E_{b}^{\prime}, c\right)=E_{b}^{\prime} \mu$. Users may also be able to set preferences to enable or disable relaying based on their current battery levels. As such, we define a utility function for the relaying terminal to be

$$
U_{R}= \begin{cases}-\infty, & \text { if } E_{r}<E_{t h} \\ R-\theta\left(E_{b}^{\prime}, c\right), & \text { otherwise }\end{cases}
$$

where $E_{r}$ is the current battery level for the potential relaying node, and $E_{t h}$ is the threshold set by the user's preferences: $E_{t h} \in(0,1]$, with a value of 1 being assigned by a user who is not interested in monetary rewards and will not cooperate in relaying.

\section{AN AUCTION BASED GAME FOR ENERGY SAVINGS}

The network operator can potentially gain additional revenue and save energy by enlisting the support of helper nodes for relaying. We propose an auction based mechanism for selecting a relaying node for a given connection.

\section{A. Auction Mechanism}

An auction is a negotiation mechanism or a set of trading rules for exchange of goods between sellers and buyers. Many auctioning mechanisms have been studied by the economics community under various conditions and mechanism rules. These various auctioning mechanisms lead to a variety of properties and outcomes of the bidding process. Some important properties to be specified in an auction are: (i) Open versus sealed-bid (bids are known or not); (ii) number of bids allowed (one time bidding versus successive bidding), (iii) winning rules, and (iv) payment (e.g., highest price, second highest price, etc.).

There is a rich literature of applying auctioning strategies for resource allocation problems in ad hoc networks. Some recent results address auction design for cooperative relaying with a solution based on best response bid updates for an SNR and a power auction solution, respectively [14].

For the case of dynamic resource allocation problems, one time bidding with sealed-bids reduces drastically the amount of information needed for bidding, and hence reduces overhead and resource allocation delays.

For these kind of applications, the Vickrey auction [15] is an appealing choice, as it has been shown to have desirable properties such as a dominant equilibrium strategy for the bidders to bid their true valuation of the goods. In a Vickrey auction, the highest bid wins, but pays the second-highest price.

For our relaying allocation problem, we consider a more complex two-dimensional bidding, in which bidders specify the estimated energy gains they provide for the base station, and their monetary reward requests. As such, a bidder $i$ will specify its bid as $s_{i}=\left(\Delta \hat{E}_{b}(i), R(i)\right)$.

The base station receives all the bids from all users and computes its utility gain from selecting node $j$ as

$$
\Delta U_{\mathrm{BS}}(j)=\Delta \hat{E}_{b}(j) c-R(j) .
$$

The base station considers for final selection only those bids for which its utility gain (6) is positive. The base station's utility gain is maximized by selecting an auction winner node $j^{*}$, such that

$$
\begin{aligned}
j^{*} & =\left.\arg \max _{j} \Delta U_{\mathrm{BS}}(j)\right|_{\Delta U_{\mathrm{BS}}(j)>0} \\
& =\left.\arg \max _{j} \Delta U_{\mathrm{BS}}(j)\left\{\Delta \hat{E}_{b}(j) c-R^{*}(j)\right\}\right|_{\Delta U_{\mathrm{BS}}(j)>0} .
\end{aligned}
$$

As we have mentioned previously, a Vickrey auction implementation is preferable, due of its desirable equilibrium property: All users have a weakly dominant strategy of bidding their own valuation of the resources.

For the Vickrey auction implementation, the equilibrium strategy for an arbitrary bidder $j$ is to select its reward $R(j)$ to be

$$
R(j)=\theta(j) .
$$

We note however that $R^{*}(j)$ in (7) does not represent the reward price claimed by user $j$, but rather, according to the rules of the Vickrey auction, the reward claimed by the user with the next lowest reward claim (second lowest ranking starting with the winner).

Theorem 1. For the proposed second price Vickrey auction with two-dimensional bids, paying the second lowest price for the winner may lead to gains less than the second highest utility gain. Furthermore, the auction pricing strategy does not guarantee positive utility gains for the base station. 
Proof: Consider user $j$ to be the winner of the auction, user $i$ to be a user that has requested the next lowest price (but not necessarily yielding the next highest gain) and user $k$ that yields the second highest utility gain for the base station.

Since node $k$ yields the second highest utility gain, we have

$$
\begin{aligned}
& \Delta E_{b}(j) c-R(j)>\Delta E_{b}(k) c-R(k) \\
\Rightarrow & \Delta E_{b}(j) c-R(i)>\Delta E_{b}(k) c-R(k)+\underbrace{(R(j)-R(i))}_{<0} .
\end{aligned}
$$

We can see that depending on the difference between the first and second utility gains for the base station and first and second lowest price requests, the base station may end up with a lower than second highest utility gain.

Further, consider the maximization of the utility for user $j$ :

$$
\begin{aligned}
& \Delta E_{b}(j) c-R(j)=\Delta E_{b}(j) c-R(i)-R(j)+R(i)>0 \\
\Rightarrow & \Delta U_{b}=\Delta E_{b}(j) c-R(i)>\underbrace{(R(j)-R(i))}_{<0} .
\end{aligned}
$$

The final base station gain is not guaranteed to be positive.

Based on the above theorem, we see that the Vickrey auction implementation is not an appealing strategy for the base station operators. We propose an alternate strategy for rewarding users, a modified Vickrey auction, for which the payment for the winner node is the requested reward plus the difference between the first and second highest utility gains.

Theorem 2. For the proposed modified Vickrey auction with two-dimensional bids, paying the winner the above pricing scheme yields an effective gain for the BS that is equal to the second highest utility gain for the bidding.

Proof: Consider user $j$ to be the winner of the auction, and user $k$ to be the node that yields the second highest utility gain for the base station. Denote by $x$ the difference between the highest and second highest utility gains. Then,

$$
\begin{aligned}
\Delta U_{b}= & \Delta E_{b}(j) c-R(j)-x \\
= & \Delta E_{b}(j) c-R(j)-\Delta E_{b}(j) c \\
& +R(j)+\Delta E_{b}(k) c-R(k) \\
= & \Delta E_{b}(k) c-R(k) \\
= & \Delta E_{b}(k) c-R(k) .
\end{aligned}
$$

Theorem 3. For the proposed modified Vickrey auction with two-dimensional bids, the above described pricing strategy ensures that all users have a dominant strategy of bidding their true valuation of resources.

Proof: The first component of the two-dimensional bid for the players comprises the users' estimates of the energy gains they can provide for the base station. For this component, the users cannot cheat, as this is the fixed resource they are selling to the BS. For the second component, pricing, users may potentially have incentives to cheat to obtain greater utilities.

Assume that node $j$ tries to cheat and departs from its true valuation bidding. Denote the node's true valuation by $\theta(j)$.

Suppose that the node decides to bid $R(j)=\theta(j)+y$.
We consider the two possible cases:

a) Node $j$ loses the auction, which results in $U_{R}(j)=0$. If the node would have won the auction by bidding $R(j)=$ $\theta(j)$ then its utility would bave been $U_{R}(j)=\theta(j)+x$, $x>0$. So overbidding decreases its utility. If bidding the true valuation would not have won the auction, then $U_{R}(j)$ remains the same which implies that there is no incentive to overbid.

b) Node $j$ wins the auction.

In this case the node gets paid $R(j)=\theta(j)+x^{\prime}$, where $x^{\prime}$ is the difference between its utility gain and the next highest one (for node $k$ ). If node $j$ would have won with a reduced price $R(j)=\theta(j)$ as well, the reward would have been $R(j)=\theta(j)+x$. Given that the strategies of other users stay the same, we have

$$
x^{\prime}=\Delta E_{b}(j) c-\theta(j)-y-\Delta E_{b}(k) c-R(k)=x-y
$$

which implies that node $j$ gets a reward of $R(j)=\theta(j)+$ $y+x-y=\theta(j)+x$ and in turn that node $j$ cannot increase its utility by raising its price above its true valuation.

Suppose that node $j$ decides to underbid its true valuation: Say, $R(j)=\theta(j)-y$.

We again consider two possible cases:

a) Node $j$ loses the auction so that $U_{R}(j)=0$, which implies that the lower bid does not change the outcome of the auction, and hence node $j$ does not have an incentive to underbid its true valuation of resources.

b) Node $j$ wins the auction.

If the action of underbidding changed the outcome of the auction, then the payment: $R(j)=\theta(j)-y+x^{\prime}$, where $x^{\prime}$ is the difference between node $j$ 's utility gain and the next highest one.

If node $j$ would have bid its true valuation, then the difference in the utility gain for the BS between the winning node $k$ and node $j$ would have been $x$.

If node $j$ 's action changed the outcome of the auction in its favor (i.e., node $j$ wins) by underbidding, then node $j$ gets as a reward $R(j)=\theta(j)-y+x^{\prime}$, where $x^{\prime}$ can be determined as

$$
x^{\prime}=\Delta E_{b}(j) c-R(j)+y-\Delta E_{b}(k) c-R(k)=y-x
$$

with $x=\Delta E_{b}(k) c-R(k)-\left(\Delta E_{b}(j) c-R(j)\right)>0$. Then, the actual reward for node $j$ can be determined to be

$$
R(j)=\theta(j)-y+y-x<\theta(j) \Rightarrow U_{R}(j)<0 .
$$

Similarly, if underbidding wins the auction, but bidding true valuation would also have won, the node utility is $U_{R}(j)=$ $x^{\prime}-y$, with

$$
x^{\prime}=\Delta E_{b}(j) c-R(j)+y-\Delta E_{b}(k) c-R(k)=y+x
$$

which implies $U_{R}(j)=x$, which does not increase with underbidding and thus node $j$ does not have an incentive to underbid its true valuation of resources. 


\section{B. Effective Green Energy Savings}

While the above proposed auctioning mechanisms ensure that both BS operators and terminals experience utility gains, we need to show under what conditions these individual utility gains result in real overall energy savings and are not merely a shift of energy consumption from the base station to the mobiles.

Assume that the true valuation of an arbitrary mobile node $j$ for its energy expenditure can be captured by a price per unit energy parameter $\mu(j)$, such that $\theta(j)=E_{b}^{\prime}(j) \mu(j)$.

In order to achieve overall energy savings, when a node $j$ wins the auction, we need to satisfy the following condition:

$$
\Delta E_{b}(j)-E_{b}^{\prime}(j)>0 .
$$

If node $j$ wins the auction then the base station has a positive gain in utility:

$$
\begin{aligned}
\Delta U_{b} & =\Delta E_{b}(j) c-E_{b}^{\prime}(j) \mu(j)-x>0 \\
& \Rightarrow \Delta E_{b}(j)-E_{b}^{\prime}(j) \mu(j) / c-\underbrace{x / c}_{>0}>0 \\
& \Rightarrow \Delta E_{b}(j)-E_{b}^{\prime}(j) \mu(j) / c>0 .
\end{aligned}
$$

If $\mu(j) / c>1$, or equivalently, $\mu(j)>c$, the above inequality guarantees effective green energy savings. Thus, for a given cost per unit energy resource, $c$, we require

$$
\mu(j)>\delta=c .
$$

We can define $\delta$ to be the reserve price for the relaying terminals, such that no resource is sold under this reserve price. The reserve price $\delta$ ensures true net energy savings for the system. We can also see that the above reserve price condition is common sense for the relaying nodes, which need to overcome their own energy costs to ensure net monetary gains.

\section{IMPLEMENTATION ISSUES}

The proposed auctioning mechanism ensures that overall effective energy savings can be implemented for the cellular systems by combining signal processing, with energy efficient resource management, relaying, and pricing.

Relaying has been shown in the literature to result in performance gains and/or energy savings for cellular networks (see for example [16] and the references therein).

In this paper, we assume that each link requires a certain BER (bit error rate) performance (which, in conjunction with error correcting codes, is directly linked to the probability of correct packet reception in the utility function definitions). The required BER can be mapped into an SINR target $\gamma$. For an illustrative example for $3 \mathrm{G}$ wireless systems based on CDMA technology, the achieved target SINR requirement for a general linear receiver filter is given as:

$$
\operatorname{SINR}_{i}=\frac{P_{t} h_{B i}\left(c_{i}^{T} s_{i}\right)^{2}}{\left(c_{i}^{T} c_{i}\right) \sigma^{2}+\sum_{k \neq i} P_{k} h_{k i}\left(c_{i}^{T} s_{k}\right)^{2}}=\gamma
$$

where $c_{i}$ is the receiver vector filter, $s_{i}$ is the signature sequence for user $i, h_{i j}$ is the path gain between transmitter $i$ and receiver $j$, and $\sigma^{2}$ is the noise power.
Hence, for a fixed transmission rate, the energy per bit gains that a mobile terminal can induce at the BS, are given by the power transmission gains due to link quality improvements:

$$
\Delta \hat{P}_{t}=\hat{P}_{t}-P_{t}^{d}=\gamma \frac{\left(c_{i}^{T} c_{i}\right) \sigma^{2}+\sum_{k \neq i} P_{k} h_{k i}\left(c_{i}^{T} s_{k}\right)^{2}}{h_{B i}\left(c_{i}^{T} s_{i}\right)^{2}}-P_{t}^{d} .
$$

The energy improvements $\Delta \hat{E}_{b}$ can be estimated by a potential relaying terminal by assessing $\Delta P_{t}$, based on initial knowledge of transmitted power required by the BS for the direct connection, $P_{t}^{d}$ (broadcasted by the BS with the call set-up request at the auction initiation phase), and by estimating the downlink path gain for the new transmission and the current interference level at terminal, together with the impact of possible improvements from signal processing (e.g., multiuser detection mitigating the interference).

We note that implementation of signal processing algorithms comes at the price of increased complexity, which in turn results in additional computational energy consumption, which adds to the energy expenditure for the relaying node. The performance/complexity tradeoff is captured by the computational energy $q$, which can be approximated based on the computational complexity of the algorithm [17].

The implementation of the auction mechanism consists of three phases [18]: Announcing, bidding, and permitting.

Announcing (the auction initiation phase): The BS announces that there is a need for a relaying terminal, together with broadcasting additional information on the requesting connection: The ID and location of the destination node, and the transmitted power required by the BS for the direct connection, $P_{t}^{d}$.

Bidding: The terminals estimate the potential power gain (12) they will bring for the requesting connection, and the energy consumption required to relay the packet. Based on this information the nodes construct the two-dimensional bid, and send it to the BS. We note here that a potential collision (or increased interference) problem among the bids may arise in practical implementations. This may be resolved either by allocating reserved mini-slots for bidding, or by implementing contention resolution mechanisms. For the latter case, the BS should wait for an estimated collision resolution time to ensure a fair decision based on receiving all bids.

Permitting: Upon receiving the bids from the terminals, the BS determines the winner based on the proposed auctioning mechanism, and broadcasts the winning node ID and the reward price.

We note that a Vickrey auction is an appealing choice because of its truthful bidding property, but in general, it has several practical limitations as mentioned in [19]. First of all, a Vickrey auction will not maximize the gain of the auctioneer (in this case the operator). We can see that this is also the case for our modified Vickrey auction solution, although we did show that a positive gain for the operator is guaranteed.

Given the above property, one of the potential problems to occur is the lying auctioneer [19], which may overstate the second best bid to the winning bidder unless that bidder can verify it. Some solutions proposed in [19], include the use of cryptographic electronic signatures, that will allow the auctioneer to 
present the second best bid to the winning bidder, or using an automated trusted third party auction server.

Despite the truthful bidding property which is true when users act independently, another potential problem in a Vickrey auction is collusion of the bidders, which could potentially result in a drastic reduction of revenue for the operator. However, this is unlikely to occur, especially in our proposed bidimensional bid auction, as a successful collusion would need to involve all bidders, and this would imply discovery of all nodes in the network, and further would require acquiring knowledge on their bids, which change dynamically with every bid announcement.

On a final note, we mention that some extreme cases may occur for which the proposed auctioning mechanism may not result in overall gains for the operator and the system. As an example, if all utility gains constructed based on the received bids are negative, the BS will simply not select a winner and will proceed with the connection without relaying. Another particular case that needs to be resolved is the case with only one terminal resulting in positive utility gain for the operator. In this case, a convention may be made to define a reserve price for the operator $\Xi^{*}$, such that this would be considered as the "second lowest bid" for the purpose of constructing the reward price for the winning terminal, while ensuring a positive gain for the operator.

\section{SIMULATION RESULTS}

Our theoretical analysis shows that effective energy gains can be achieved using the proposed auctioning mechanism. In this section, we are quantifying the magnitude of the achievable gains via simulations results in Matlab. We simulate a singlecell CDMA system of radius $R=2500 \mathrm{~m}$ operating in the $900 \mathrm{MHz}$ frequency band with a transmission bandwidth of $3.84 \mathrm{MHz}$ that is similar to a $3 \mathrm{G}$ system. A transmission rate of $\mathrm{R}=32 \mathrm{~K}$ is considered for data. Destination terminals are employing matched filter receivers, and the computed capacity for this cell is 22 users. The target SINR for the system is $\gamma=5.75$. Power control is implemented to ensure that all links meet target SINR.

Our simulation set-up considers a number of terminals uniformly distributed within the cell, with some active connections already in use. The number of active connections determines the load of the cell, e.g., 6 active connections is approximately a $25 \%$ load.

We investigate the energy and monetary gains achieved by the base station, as well as the effective (green) energy gains, when a new connection is set-up in a cell with a given load by using the proposed auctioning algorithm. The performance metrics are obtained by averaging over 1000 different cell realizations (in terms of nodes positions, and random selection of destination nodes).

We first investigate what is the optimum value for the terminals' unit price per energy $\mu$, in terms of multiples of $c$ (unit cost for energy).

In (10), we showed that a sufficient condition for energy gains is to have $\mu>c$, but this is not a necessary condition. We can see in Fig. 1, that overall effective energy savings can still be achieved for $\mu<c$, but they are greatly reduced, and further this

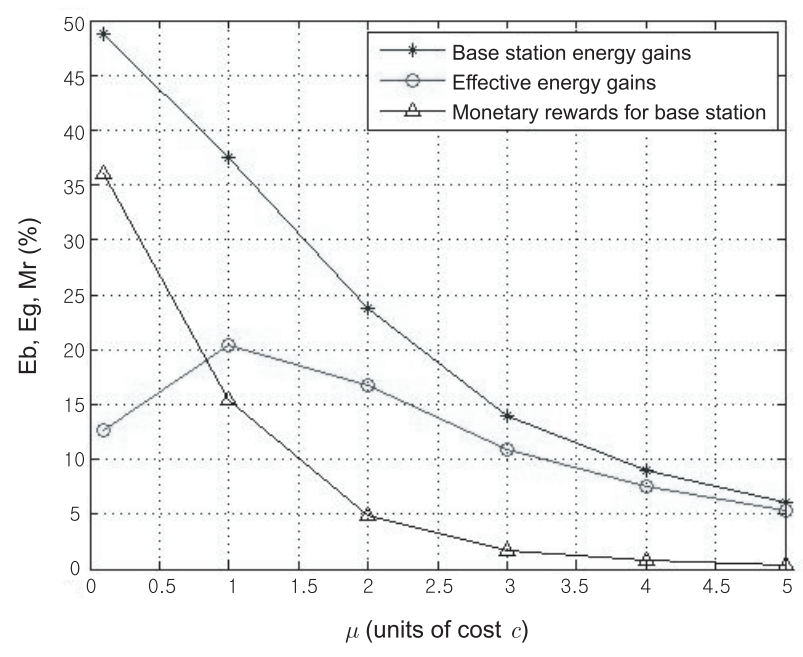

Fig. 1. Energy and monetary gains versus pricing (50\% load for the cell).

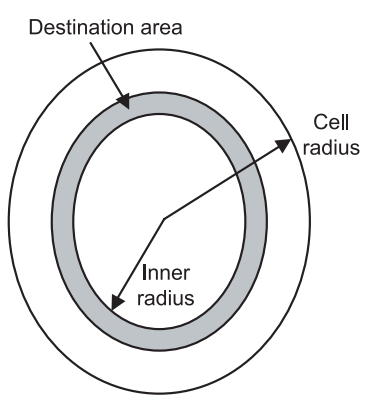

Fig. 2. Destination nodes' positions within the cell.

may not be an appealing choice for the relaying terminals. From Fig. 1, we can see that while the base station operators benefit from cheaper relaying, the effective energy gains are very small in this case, and an optimal effective energy gain is obtained for $\mu=c$. We note that the terminals are more likely to prefer $\mu>c$.

The price $\mu$ could be potentially regulated by agreement between operators and terminals. From Fig. 1, we can see that a range for $\mu$ between 1 and 1.5 would be beneficial for the overall system.

We investigate also how the energy and monetary gains vary with the load (Fig. 3), the available number of nodes for relaying (Fig. 4), and the position of the destination node within the cell (Fig. 5).

In Fig. 3, we can see that lightly loaded systems benefit more from relaying, with achieved overall energy savings up to $50 \%$. As the load increases, the system becomes more sensitive to the interference created by the additional relays, and thus the gains reduce. This illustrates the inherent tradeoff between saving transmission power for individual transmissions while adding more interferers into the system, albeit at a lower transmission power.

To study how the gains vary with the destination node's position in the cell, we position the destination nodes in a ring, with varied inner radii within the cell (Fig. 2). 


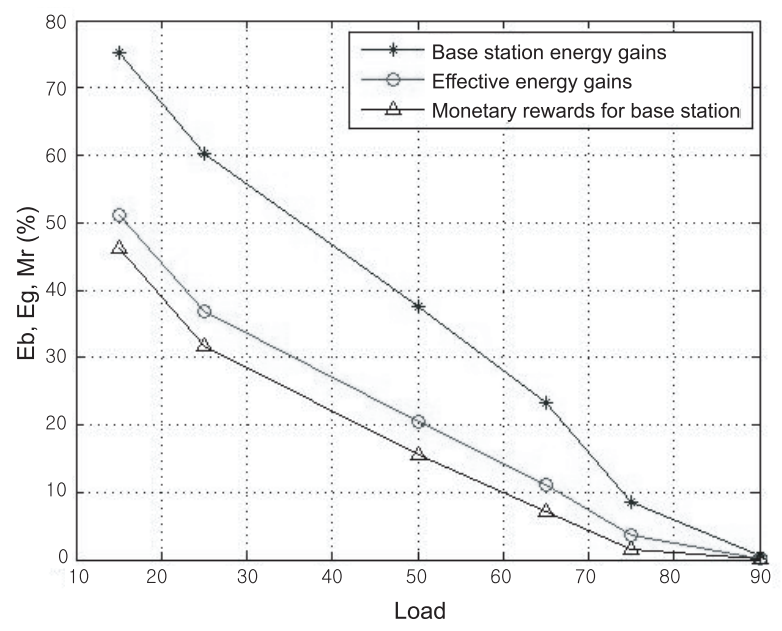

Fig. 3. Energy and monetary gains versus load (50 nodes available, outermost destination region).

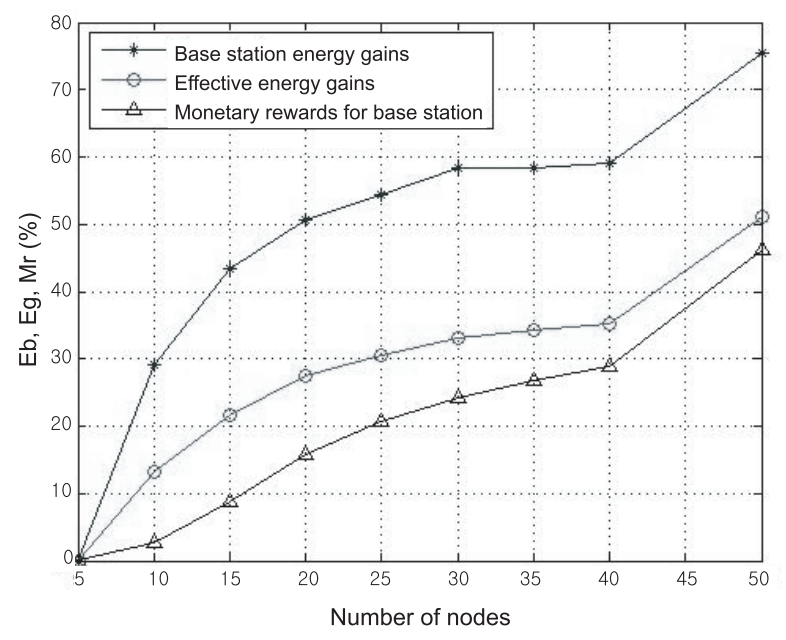

Fig. 4. Energy and monetary gains versus number of available nodes ( $25 \%$ load, outermost destination region).

We consider a destination nodes' positions ring of width $0.2 R$ ( $R=$ radius of the cell), and we vary the inner radius from $0.2 R$ to $0.8 R$ (Fig. 5).

As expected, from Fig. 5, we note that the highest energy gains are obtained when the destination nodes are at the outermost ring in the cell, which is the case that demands the most energy from the base station. We can see that in this case, we obtain overall energy gains up to $50 \%$.

To summarize, our proposed auction based relaying mechanism becomes very effective when the system is lightly loaded, a sufficient number of terminals are available for relaying (good positions for relaying terminals are available) and the direct connection is energy hungry (the destination node is positioned towards the edge of the cell).

As a final note, we mention that our simulations have made the implicit assumption that all the links used for transmission or exchanging bidding information meet their target SIR require-

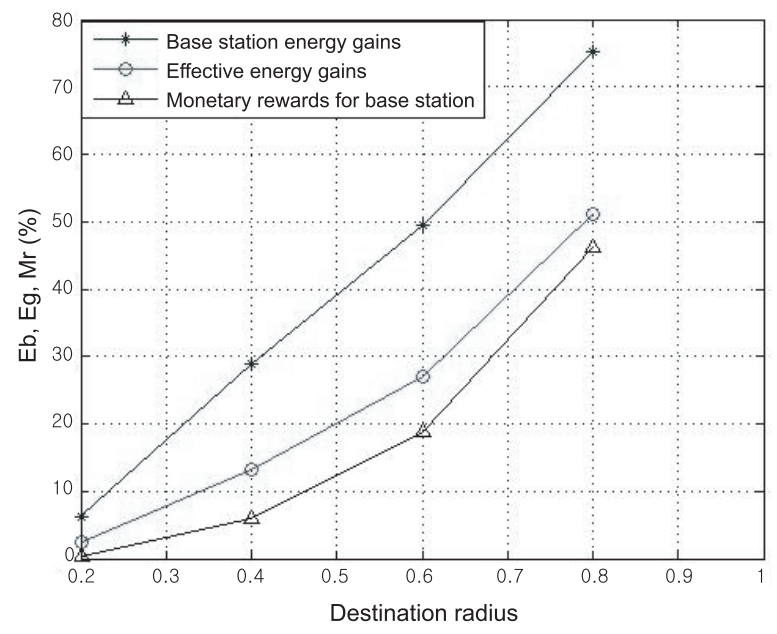

Fig. 5. Energy and monetary gains versus destination area (25\% load, $N=50$ nodes available).

ment (power control was also implemented), and such the packet error rate is very small. Future work may investigate how the system is affected if errors do affect the bidding process.

\section{CONCLUSIONS}

In this paper, we have proposed a pricing scheme and an auctioning mechanism to enforce terminals-base station cooperation for achieving effective energy gains for a cellular system. We have shown that, for the proposed auctioning strategy, network operators are guaranteed revenue gains, mobile nodes' dominant strategy is to bid their true valuation of their energy resources, and overall effective energy gains occur under the assumption of a reserve price for bidding. Our simulation results show that significant effective energy savings can be achieved (up to $50 \%$ ) by employing this auctioning mechanism for a $3 \mathrm{G}$ CDMA cellular set-up.

\section{REFERENCES}

[1] O. Naomi, "Beyond the ivory tower: The scientific consensus on climate change," 5702, s.1: Science, (Kyoto, Japan), Dec. 2004, p. 1686.

[2] J. Hansen, M. Sato, P. Kharecha, G. Russell, D. Lea, and M. Siddal, "Climate change and trace gases," Philosophical Trans. Royal Society, vol 365, pp. 1925-1954, May 2007.

[3] Green radio. Wireless@KTH. Royal Institute of Technology (KTH). [Online]. Available: http://www.wireless.kth.se/projects/GREEN/index.php.

[4] P. Grant, "Green radio - The case for more efficient cellular base stations," University of Edinburgh. [Online]. Available: http://www.see. ed.ac.uk/ pmg

[5] J. Hayes and T. Haynes, "Opinion-first person - If you ask me, is wireless set to go green?" Engineering and Technology, vol. 2, no 11, pp. 24-24, Feb. 2008 .

[6] D. Ezri and S. Shilo, "Green cellular - Optimizing the cellular network for minimal emission from mobile stations," arXiv:0906.5289v1 [cs.IT], submitted June 2009.

[7] B. Badic, T. O'Farrrell, P. Loskot, and J. He, "Energy efficient radio access architectures for green radio: Large versus small cell size deployment," in Proc. IEEE VTC, Anchorage, Alaska Sept. 2009.

[8] F. Richter, A. Fehske, and G. Fettweis, "Energy efficiency aspects of base station deployment strategies for cellular networks," in Proc. IEEE VTC, Anchorage, Alaska Sept. 2009. 
[9] V. Bassoo, K. Tom, A. K. Mustafa, E. Cijvat, H. Sjoland, M. Faulkner, "Potential architecture for future generation green wireless base station," in Proc. 4th Int. Symp. Wireless Pervasive Computing, 2009.

[10] C. Comaniciu, N. B. Mandayam, and H. V. Poor, "Radio resource management for green wireless networks," in Proc. IEEE VTC, Anchorage, Alaska Sept. 2009.

[11] H. Karl, "An overview of energy-efficiency techniques for mobile communication systems," Telecommunication Networks Group, Technical University Berlin, Tech. Rep. TKN-03-XXX, Sept. 2003.

[12] C. Saraydar, N. B. Mandayam, and D. J. Goodman, "Power control via pricing in wireless data network," IEEE Trans. Commun., vol. 50, no. 2, pp. 291-303, Feb. 2002.

[13] O. Ileri, S.-C. Mau, and N. B. Mandayam, "Pricing for enabling forwarding in self-configuring ad hoc networks," IEEE J. Sel. Areas Commun., vol. 23, No. 1, pp. 151-162, Jan. 2005.

[14] J. Huang, Z. Han, M. Chiang, and H. V. Poor, "Auction-based resource allocation for cooperative communications," IEEE J. Sel. Areas Commun., vol. 26, no. 7, pp. 1226-1237, Sept. 2008.

[15] V. Krishna, Auction Theory, London, UK: Academic Press, 2002.

[16] C. Raman, G. J. Foschini, R. A. Valenzuela, R. D. Yates, and N. B. Mandayam, "Power savings from half-duplex relaying in downlink cellular systems," in Proc. IEEE Globecom, 2009.

[17] S. M. Futaci, K. Jaffres Runser, and C. Comaniciu, "On modeling energysecurity tradeoffs for distributed monitoring in wireless ad hoc networks," in Proc. IEEE MILCOM, San Diego, CA, Nov. 2008.

[18] H. Chang, K.-C. Chen, N. Prasad, and C.-W Su, "Auction based spectrum management of cognitive radio networks," in Proc. IEEE VTC, Barcelona, Spain, 2009.

[19] T. Sandholm, "Issues in computational Vickrey auctions," Int. J. Electronic Commerce, vol. 4, no. 3, Special Isssue on Intelligent Agents for Electronic Commerce, pp. 107-129, 2000.

[20] J. Huang, R. Berry, and M. L. Honig, "Auction-based spectrum sharing," Mobile Networks and Applications, vol. 11, no. 3, pp. 405-418, June 2006.

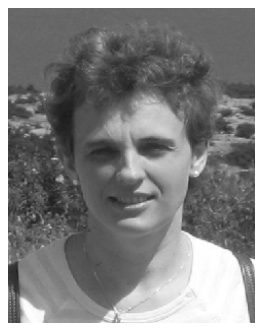

Cristina Comaniciu received the M.S. degree in Electronics from the Polytechnic University of Bucharest in 1993, and the Ph.D. degree in Electrical and Computer Engineering from Rutgers University in December 2001. From 1998 to 2001, she was with WINLAB, Rutgers University, and from 2002 to 2003 she was affiliated with the Electrical Engineering Department at Princeton University as a research associate. Since August 2003, she is with Stevens Institute of Technology, Department of Electrical and Computer Engineering, where she is now an Associate Professor. She is a recipient of the 2007 IEEE Marconi Best Paper Prize Award in Wireless Communications for a paper co-authored with $\mathrm{H}$. Vincent Poor. She currently serves as an Associate Editor for the IEEE Communications Letters. Her research interests include green radio, cooperative protocols for spectrum sharing and interference mitigation, cross-layer design, game theoretic approaches for design of energy aware wireless networks, radio resource management for cellular and ad hoc networks, and energy-security tradeoffs for wireless networks.

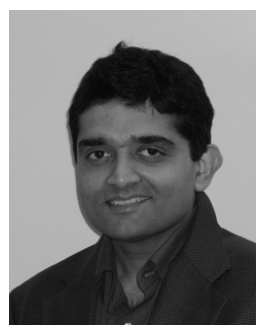

Narayan B. Mandayam received the B.Tech. (Hons.) degree in 1989 from the Indian Institute of Technology, Kharagpur, and the M.S. and Ph.D. degrees in 1991 and 1994 from Rice University, all in Electrical Engineering. From 1994 to 1996, he was a Research Associate at the Wireless Information Network Laboratory (WINLAB), Rutgers University before joining the faculty of the Electrical and Computer Engineering Department at Rutgers where he became Associate Professor in 2001 and Professor in 2003. Currently, he also serves as Associate Director at WINLAB. He was a visiting faculty fellow in the Department of Electrical Engineering, Princeton University in 2002 and a visiting faculty at the Indian Institute of Science in 2003. His research interests are in various aspects of wireless data transmission including system modeling and performance, signal processing and radio resource management with emphasis on techniques for cognitive radio networks. $\mathrm{He}$ is a recipient of the Fred W. Ellersick Prize from the IEEE Communications Society in 2009 along with O. Ileri for their work on dynamic spectrum access models and spectrum policy. He is also a recipient of the Institute Silver Meda from the Indian Institute of Technology in 1989 and the National Science Foundation CAREER Award in 1998. He is a coauthor with C. Comaniciu and $\mathrm{H}$. V. Poor of the book "Wireless Networks: Multiuser Detection in Cross-Layer Design," Springer, NY. He has served as an Editor for the journals IEEE Communication Letters and IEEE Transactions on Wireless Communications. He has also served as a guest editor of the IEEE Journal on Selected Areas in Communications Special Issues on Adaptive, Spectrum Agile and Cognitive Radio Networks (2007) and Game Theory in Communication Systems (2008). He is a Fellow of the IEEE.

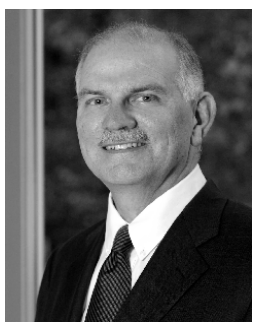

H. Vincent Poor received the Ph.D. degree in EECS from Princeton University in 1977. From 1977 until 1990, he was on the faculty of the University of Illinois at Urbana-Champaign. Since 1990 he has been on the faculty at Princeton, where he is the Michael Henry Strater University Professor of Electrical Engineering and Dean of the School of Engineering and Applied Science. His research interests are in the areas of stochastic analysis, statistical signal processing and information theory, and their applications in wireless networks and related fields. Among his publications in these areas are the recent books Quickest Detection (Cambridge University Press, 2009) and Information Theoretic Security (Now Publishers, 2009).

$\mathrm{He}$ is a member of the U. S. National Academy of Engineering, a Fellow of the American Academy of Arts and Sciences, and an International Fellow of the Royal Academy of Engineering (U. K). He is also a Fellow of the IEEE, the Institute of Mathematical Statistics, and other organizations. In 1990, he served as President of the IEEE Information Theory Society, and in 2004-07 he served as the Editor-in-Chief of the IEEE Transactions on Information Theory. $\mathrm{He}$ is the recipient of the 2005 IEEE Education Medal. Recent recognition of his work includes the 2007 Technical Achievement Award of the IEEE Signal Processing Society, the 2008 Aaron D. Wyner Distinguished Service Award of the IEEE Information Theory Society, and the 2009 Edwin Howard Armstrong Achievement Award of the IEEE Communications Society.

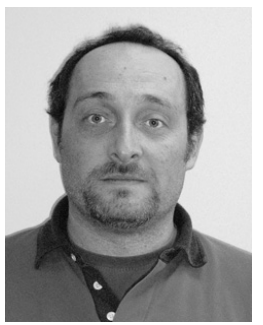

Jean-Marie Gorce received the M.Sc. and Ph.D. degrees in 1993 and 1998, respectively. He is a Professor of Wireless Communications at the National Institute of Applied Sciences (INSA), Lyon, France. He became the Director of CITI (Centre for Innovation in Telecommunications and Integration of Services) in September 2009. Since 2001 he is a member of INRIA (French National Institute for Research in Computer Science and Control) the most prestigious French research institute in Computer sciences ans since January, 2009, he is the leader of the team Swing (Smart Wireless Networking). He is also a member of the ADR Selfnet, a join lab between INRIA and Alcatel Lucent. Since 2001, he was involved as a leading scientist in several French and European projects. His main research fields concern wireless communications focusing on realistic modeling, wireless system optimization and performance assessment considering both infrastructurebased and ad hoc networks. He has published over 70 refereed journal and conference papers and currently serves as Associate Editor of Telecommunication Systems and Eurasip Journal on Wireless Communications and Networking. 